Light output of Double Chooz scintillators for low energy electrons

This article has been downloaded from IOPscience. Please scroll down to see the full text article.

2011 JINST 6 P11006

(http://iopscience.iop.org/1748-0221/6/11/P11006)

View the table of contents for this issue, or go to the journal homepage for more

Download details:

IP Address: 149.217.1.5

The article was downloaded on 22/11/2011 at 09:38

Please note that terms and conditions apply. 


\title{
Light output of Double Chooz scintillators for low energy electrons
}

\author{
C. Aberle, C. Buck, F.X. Hartmann, ${ }^{1}$ S. Schönert ${ }^{2}$ and S. Wagner ${ }^{3}$ \\ Max-Planck-Institut für Kernphysik, \\ Saupfercheckweg 1, 69117 Heidelberg, Germany \\ E-mail: Stefan.Wagner@mpi-hd.mpg.de
}

\begin{abstract}
The energy dependent light output of liquid scintillators used in the Double Chooz experiment was measured for electrons up to $140 \mathrm{keV}$ energy. A new Compton scattering coincidence apparatus was built for this purpose. A detailed study on possible systematic errors was made. We report the experimental results of our investigations and tested them for concordance with the predictions of various models. All models reasonably fit the experimental data after adjusting the respective free parameters. The results were also used to tune the Geant4-based Monte Carlo simulation software which is used in Double Chooz. The experimental data can be described by the simulation choosing an effective value for the Birks parameter.
\end{abstract}

KEYWORDS: Scintillators, scintillation and light emission processes (solid, gas and liquid scintillators); Liquid detectors; Detector modelling and simulations I (interaction of radiation with matter, interaction of photons with matter, interaction of hadrons with matter, etc); Large detector systems for particle and astroparticle physics

\footnotetext{
${ }^{1}$ Current address: Hartmann Scientific, City of Virginia Beach, Virginia, U.S.A.

${ }^{2}$ Now at Technische Universität München.

${ }^{3}$ Corresponding author.
} 


\section{Contents}

1 Introduction 1

2 Experimental 3

$\begin{array}{llr}3 & \text { Investigation of systematic errors } & 8\end{array}$

4 Results 9

5 Monte Carlo tuning $\quad 14$

6 Conclusion 17

\section{Introduction}

The Double Chooz experiment (DC) aims to measure the electron antineutrino flux from two nearby nuclear reactors with two identical detectors at different distances from the reactors [1]. In the neutrino oscillation framework a deficit of the neutrino flux in the far detector can be used to determine the mixing angle $\theta_{13}$. Each detector makes use of three different scintillator mixtures: a gadolinium-loaded Neutrino Target ( $v$-Target) for the $\bar{v}_{e}$-detection, a Gamma Catcher (GC) for a full energy deposition in the active volume of the detector, and a Muon Veto ( $\mu$-Veto). Additionally, a non-scintillating buffer liquid is used to shield the inner two volumes from radioactivity.

In Double Chooz the channel for neutrino detection is the antineutrino capture on hydrogen $\bar{v}_{e}+p \rightarrow n+e^{+}$. The signature of this event comprises a prompt and a delayed signal. The prompt signal is caused by the positron traversing the scintillator and its subsequent annihilation. The neutron from the reaction first thermalizes and is then captured on a gadolinium nucleus with a time constant of about $30 \mu \mathrm{s}$. The subsequent deexcitation of the nucleus creates gammas of $8 \mathrm{MeV}$ total energy.

The scintillators for the $v$-Target and Gamma Catcher have been developed and produced at the Max-Planck-Institut für Kernphysik (MPIK) in the framework of the research and development for novel metal loaded liquid scintillators [2-6]. Details of the development and production of the scintillators discussed here will be given in [7]. As the preceding experiment Chooz was limited by the lifetime of its scintillators, long-term stability was the key requirement for the DC scintillators. Additionally, the scintillators have to be radiopure, chemically compatible with the acrylic tanks, and furthermore provide high transparency and scintillation performance. These requirements led to the development of a novel gadolinium-loaded $v$-Target scintillator [5, 7]. An overview over the scintillators' chemical composition is given in table 1. 
Table 1. Composition of the liquid scintillators used in DC.

\begin{tabular}{|c|c|}
\hline Scintillator & Composition \\
\hline \multirow[t]{6}{*}{$v$-Target } & $80 \%{ }_{\text {vol }}$ n-dodecane \\
\hline & $20 \%_{\text {vol }}$ PXE $^{1}$ \\
\hline & $4.5 \mathrm{~g} / \mathrm{l} \mathrm{Gd}-(\mathrm{thd})_{3}{ }^{2}$ \\
\hline & $7 \mathrm{~g} / 1 \mathrm{PPO}^{3}$ \\
\hline & 20 mg/l bis-MSB ${ }^{4}$ \\
\hline & $0.5 \%_{\mathrm{wt}}$ tetrahydrofuran \\
\hline \multirow[t]{5}{*}{ Gamma Catcher } & $66 \% \%_{\text {vol }}$ Shell Ondina $909^{5}$ \\
\hline & $30 \%_{\text {vol }}$ n-dodecane \\
\hline & $4 \%_{\mathrm{vol}} \mathrm{PXE}$ \\
\hline & 2 g/l PPO \\
\hline & 20 mg/l bis-MSB \\
\hline \multirow[t]{4}{*}{$\mu$-Veto } & $62.5 \%$ tetradecane \\
\hline & $37.5 \% \mathrm{LAB}^{6}$ \\
\hline & $3 \mathrm{~g} / \mathrm{lPPO}$ \\
\hline & 20 mg/l bis-MSB \\
\hline
\end{tabular}

Special importance, when using liquid scintillators, has to be given to ionization quenching. Following the definition in [8], this is the loss of scintillation light "attributed to quenching of the primary excitation by the high density of ionized and excited molecules". It is strong in regions where the specific energy loss $d E / d x$ of the primary particle is large, i.e. towards the end of the particle track or for particles of low energies. The specific energy loss is consequently the main quantity in the different ionization quenching models (see section 4). Throughout this article the term quenching will refer to this specific type of light loss.

The investigation of quenching is of importance for a precise energy reconstruction of scintillation events. An accurate reconstruction demands an appropriate model for the response function $L(E)$ - the amount of scintillation light emitted for an incident particle of initial energy $E$. While $L(E)$ is linear for higher energies, the effect of quenching increases with decreasing energy of the incident particle, and $L(E)$ becomes non-linear for small $E$. In the case of single electrons, the deviation from linearity is significant below ca. $100 \mathrm{keV}$; for heavier particles (alphas, protons, etc.) non-linearities have to be considered up to the $\mathrm{MeV}$ scale [8]. In Double Chooz the total energy of the $\mathrm{Gd}$ deexcitation is about $8 \mathrm{MeV}$, but it is released in several gamma rays, which each deposit their energies in multiple Compton scatter events and numerous electrons of lower energies are created. Each low-energy electron is then affected individually by quenching, which makes the strength of the total effect considerable even for MeV-events.

\footnotetext{
${ }^{1}$ Ortho-phenylxylylethane; CAS: 6196-95-8.

${ }^{2}$ Gd(III)-tris-(2,2,6,6-tetramethyl-heptane-3,5-dionate); CAS: 14768-15-1.

3 2,5-diphenyloxazole; CAS: 92-71-7.

4 4-bis-(2-methylstyryl)benzene; CAS: 13280-61-0.

${ }^{5}$ CAS: 8042-47-5.

${ }^{6}$ Linear alkyl benzene; CAS: 27176-87-0.
} 


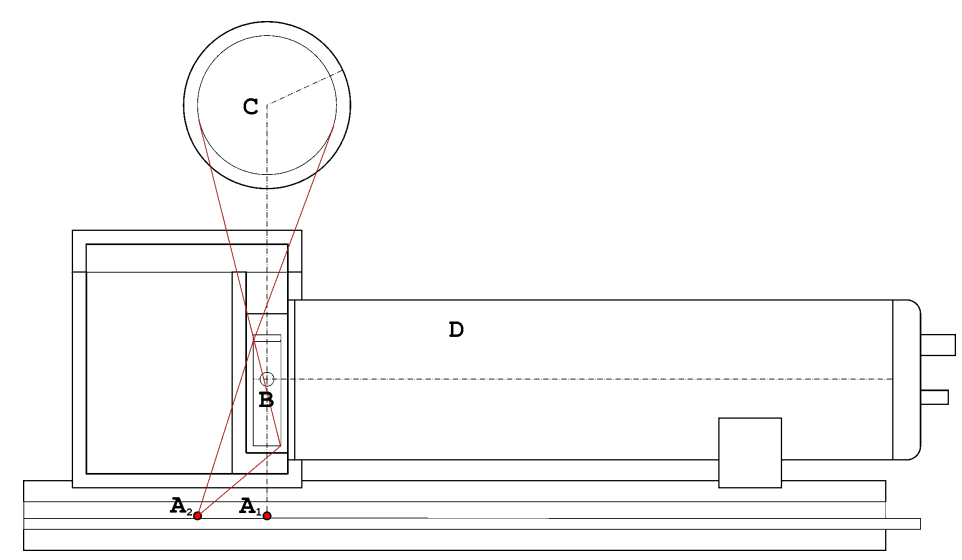

Figure 1. Schematic view of the experimental setup. Main parts are the ${ }^{137} \mathrm{Cs}$ sources $\left(\mathrm{A}_{1}\right)$ and $\left(\mathrm{A}_{2}\right)$, the scintillator cell (B), the germanium spectrometer (C) and the PMT (D). The smallest and largest scattering angle possible for the second source are also drawn.

\section{Experimental}

The scintillator light output $L\left(E_{e}\right)$ for different electron energies $E_{e}$ was measured using monoenergetic gamma rays of energy $E_{\gamma}$ undergoing Compton scattering off electrons in the scintillator sample. The Compton electron released creates light as it traverses the scintillator. A PMT observes the scintillation, while the energy of the scattered photon $E_{\gamma}^{\prime}$ is directly measured with a $0.83 \mathrm{~kg}$ high-purity germanium spectrometer (HPGe) located above the sample. The energy of the Compton electron $E_{e}$ can then be obtained via $E_{e}=E_{\gamma}-E_{\gamma}^{\prime}$. With the requirement of coincidence of the two signals a scintillation pulse can be related to a specific electron energy. A similar setup has been used successfully in [9]. An advantage of this technique is that the electrons are created directly within the liquid scintillator. This avoids surface effects which may occur if one uses external incident electrons. In comparison to a measurement of the scattering angle, the precise determination of the photon energy in a HPGe-spectrometer is independent of the exact setup geometry or the finite sample size. This allows for a compact arrangement and drastically reduces measurement times for a given source activity.

Experimental setup. Our setup is illustrated schematically in figure 1. The central component is a fused quartz vial of $10 \mathrm{~mm} \times 10 \mathrm{~mm} \times 35 \mathrm{~mm}$ inner dimensions. The vial is first cleaned with cyclohexane and left to dry in an oven. Then the scintillator sample is filled and bubbled with nitrogen. Afterwards, the vial is tightly sealed with a teflon cap and vacuum putty. For light protection it is placed in a blackbox, which provides a fused quartz window for the PMT but is otherwise light-tight. The sample is irradiated by gamma rays from two point-like radioactive sources below the vial. The $\gamma$-emitters in use are two ${ }^{137} \mathrm{Cs}$ sources of ca. $400 \mathrm{kBq}$ and $200 \mathrm{kBq}$ activity, which emit monoenergetic photons of $662 \mathrm{keV}$ energy. Since the apparatus only records events in which a photon is deflected into the direction of the HPGe-spectrometer (because of the coincidence requirement) the sources were positioned such that scattering angles between $0^{\circ}$ and about $45^{\circ}$ could be detected, corresponding to about $0-180 \mathrm{keV}$ electron energy in the scintillator. When a Compton electron is created within the sample, its scintillation is observed by a Photonis XP2262 photomultiplier tube. The energy of the scattered photon is directly measured with a 


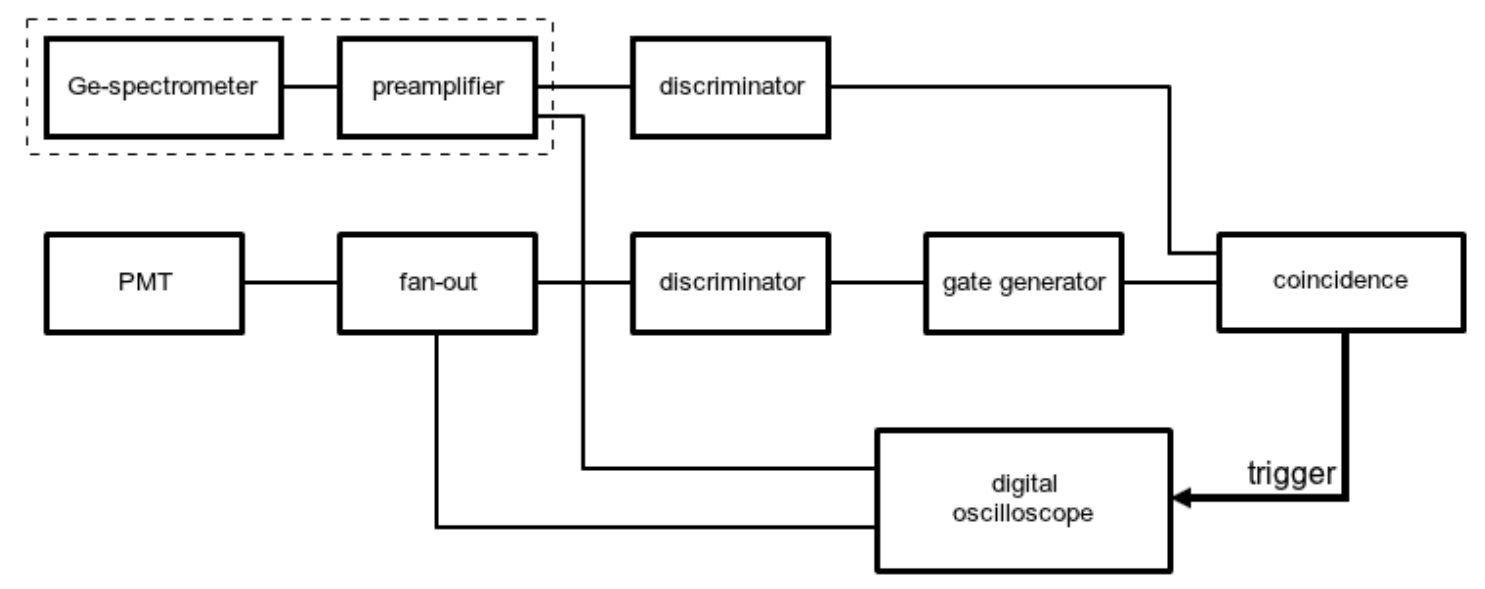

Figure 2. Schematic of the electronic circuitry used in the experiment.

HPGe-spectrometer by Princeton Gamma-Tech. To reduce background radiation, the experiment was conducted at the underground low-level laboratory at the MPIK. It provides an overburden of $15 \mathrm{~m}$ w.e. against the hadronic component of cosmic radiation. The whole setup is further shielded from radiation by lead bricks.

Data acquisition. The data acquisition chain is depicted in figure 2. The PMT signal is duplicated in a LeCroy $428 \mathrm{~F}$ fan-in/fan-out module. One of the output signals is used to create the coincidence, the other one is fed into a Tektronix TDS 7054 DPO oscilloscope. The spectrometer's charge sensitive preamplifier already provides two output channels of which one directly enters the oscilloscope and the other is used to create the coincidence signal. For this purpose the PMT- and HPGe-signals run through a PS 730 discriminator module and are converted into logical pulses. The (logical) PMT signal is fed into an Ortec 416A gate generator to open a gate. Its width is equivalent to the coincidence time $T_{\text {coinc }}$, which, after an analysis of the distribution of the time differences between the signals, has been set to $500 \mathrm{~ns}$. Finally, the gate output and the (logical) HPGe-signal enter a LeCroy 465 coincidence module. The coincidence output then triggers the oscilloscope, which simultaneously records the signals from the PMT and the HPGe-detector's preamplifier output and writes them to disk for the off-line analysis. The horizontal time resolution was set to $0.4 \mathrm{~ns}$ per channel and the record length adjusted to 10000 channels, corresponding to $4 \mu \mathrm{s}$. The vertical resolution corresponds to 256 channels (ADC counts) and the voltage scales were set to $1000 \mathrm{mV}$ for HPGe-pulses and $2000 \mathrm{mV}$ for the PMT pulses.

Data analysis. The files are read out by a program which extracts the electron energies and the corresponding scintillation light yield. An event-wise calculation of the baseline is performed for both signals. The light output is obtained by a numerical integration over the baseline-corrected PMT pulse. The HPGe-signal is first processed by a pulse shaping algorithm to reduce the effect of statistical fluctuations on the maximum value. A moving average filter of $28 \mathrm{~ns}$ window width is passed over the pulse, which calculates the mean value of adjacent bins. The pulse height is given as the difference between the maximum value and the baseline. It is then converted into the deposited gamma energy $E_{\gamma}$ with data available from the spectrometer calibration (section 3). The 


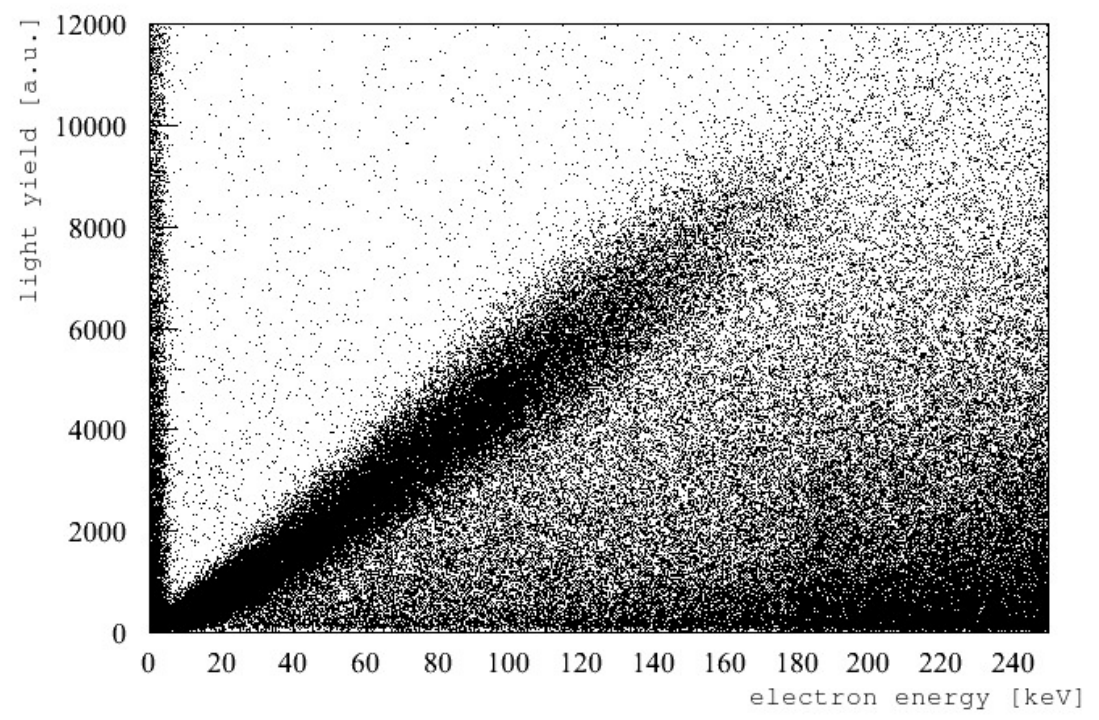

Figure 3. Typical distribution of obtained light yields vs. the electron energy deposited in the scintillator. The dense cloud of events contains the response curve.

energy of the Compton electron in the sample is the difference between $E_{\gamma}$ and the source energy. In addition, the program rejects "defective" events, e.g. such with double pulses or baseline jitter.

The scintillation light is then plotted versus the electron energies. Figure 3 shows a typical scatter plot. The dense area along the diagonal are events in which the gamma energy is shared between the liquid scintillator and the HPGe-detector. The other events are background. To the right of the dense cloud are entries caused by multiple Compton scattering, where the scattered photon does not deposit all its energy in the germanium crystal. The electron energy is therefore calculated higher than it is in reality. The sparser area on the left is populated with two-photon coincidences, where the narrow band occurs when one photon undergoes photoabsorption in the spectrometer.

To obtain a "clean" $L(E)$, the data is divided into narrow intervals $\left[E_{1}, E_{2}\right]$ of electron energies. The distribution of the light output of the events within this interval is projected into a new histogram. Figure 4 shows such a "slice" for $E_{e}=59-61 \mathrm{keV}$. A width of $2 \mathrm{keV}$ for each interval has been chosen. A slice can be represented by a vertical cross section of the distribution in figure 3 at a specific $E_{e}$. The electron energy attributed to the slice is the mean energy of the events in it.

The events in the left part of figure 4 are background events and are modeled by an exponential function. The information about the scintillator response is contained in the peak to the right. According to the working principle of a PMT, its shape is the result of a convolution of a Poisson distribution with Gaussian functions. Nevertheless, our investigations have shown that the peak can be fit more efficiently by a Gaussian with only a negligible error to the position of the maximum, which corresponds to $L\left(E_{\text {slice }}\right)$. The fit function used was the sum of an exponential and a Gaussian function. The leftmost part of the spectrum was not included in the fit to avoid an influence of the discriminator threshold on the fit results. Finally the values for $L\left(E_{\text {slice }}\right)$ were plotted against $E_{\text {slice }}$. A plot of the response function is given in figure 5. Figure 6 shows the deviation of the data points from a perfectly linear behavior. It is obtained by defining a proportional function $f(E)=a \cdot E$ 


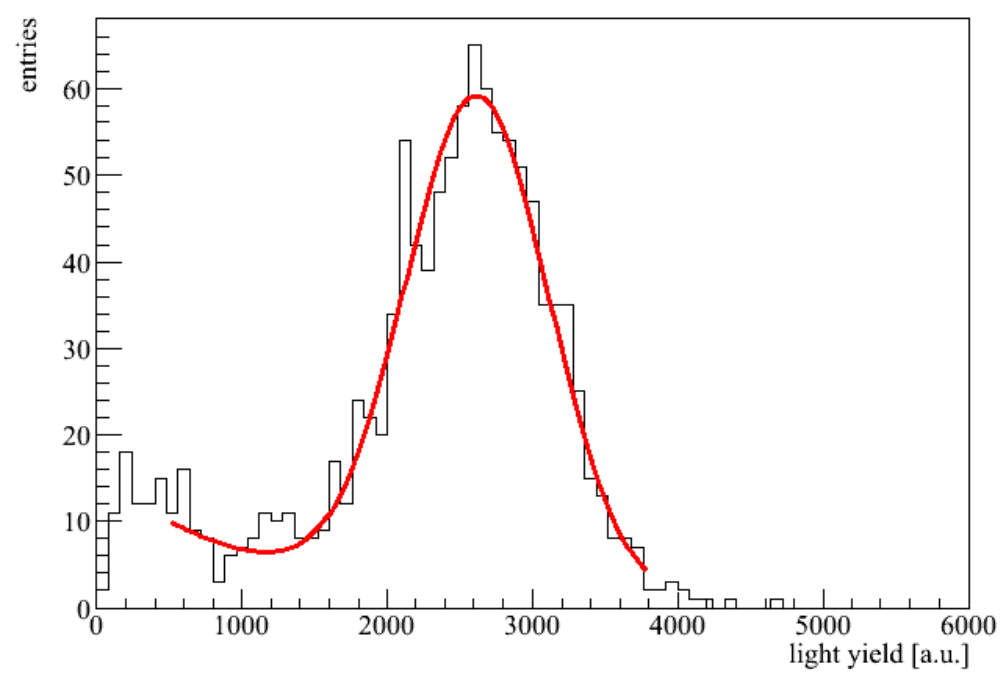

Figure 4. Energy slice of 59-61 keV electron energy as obtained from figure 3. The mean value of the Gaussian is the light yield response to electrons of that energy; the entries to the left are background. The function used to fit the histogram is the sum of an exponential and a Gaussian.

through a reference point at $139 \mathrm{keV}$, and then plotting the relative difference of the experimental data points to this function. As the reference point can be chosen freely the resulting curve may exceed 1 .

The following scintillator samples were measured:

1. $v$-Target: the DC $v$-Target scintillator was measured with a composition as in table 1 .

2. Gamma Catcher: the DC Gamma Catcher was studied in two versions, once with the composition described in table 1 and in a modified version with $5 \mathrm{~g} / \mathrm{lPPO}$. The second composition was under discussion as a backup option for the experiment.

3. $\mu$-Veto: the sample measured was a candidate for the DC $\mu$-Veto and is described in table 1 . The final version has a different composition, notably with more LAB and less PPO.

4. Borexino: we also measured the scintillator used in the Borexino experiment, consisting of $1.5 \mathrm{~g} / 1 \mathrm{PPO}$ dissolved in PC ${ }^{7}$. This sample offered a good opportunity to study a scintillator of relatively simple composition. It also serves as a cross-check, as information on PC-based scintillators is readily available, e.g. in [9].

5. Plastic scintillator: the last sample is a commercial polystyrene-based plastic scintillator with p-terphenyl and $\mathrm{POPOP}^{8}$ as fluors. This material was provided by the CALICE-group at the Universität Heidelberg and has been measured to study differences between liquid and plastic scintillators.

\footnotetext{
${ }^{7}$ Pseudocumene (1,2,4-trimethylbenzene).

8 1,4-bis(5-phenyloxazol-2-yl) benzene, CAS: 1806-34-4.
} 


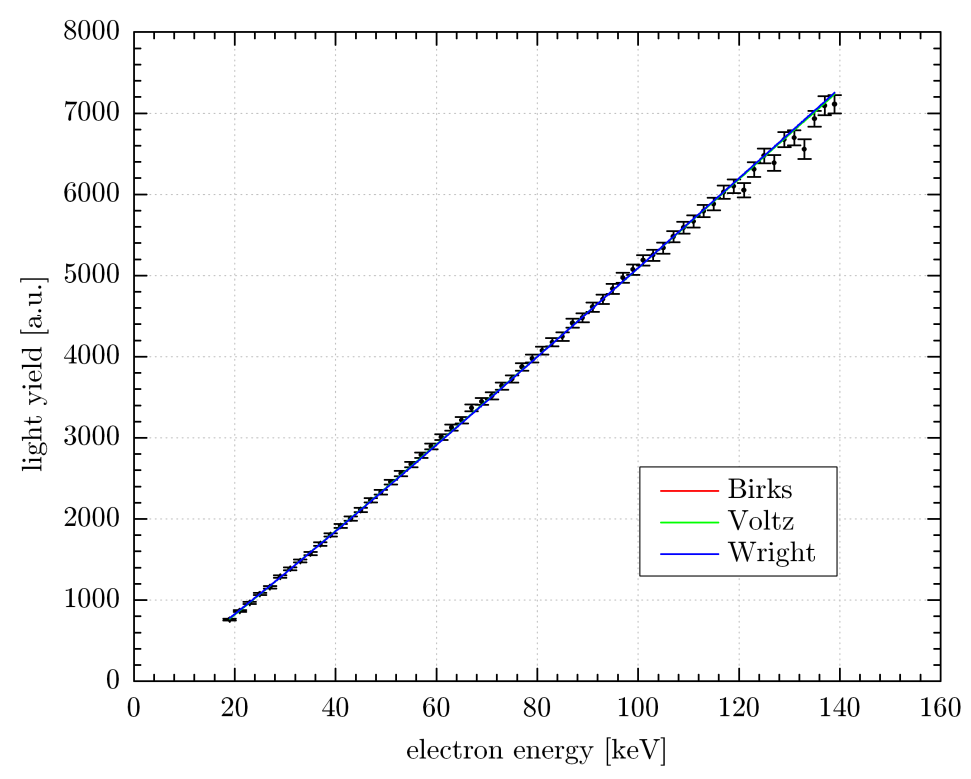

Figure 5. Light yield data points obtained from figure 3 after the slice fitting procedure. The plot shows the results for the $v$-Target scintillator.

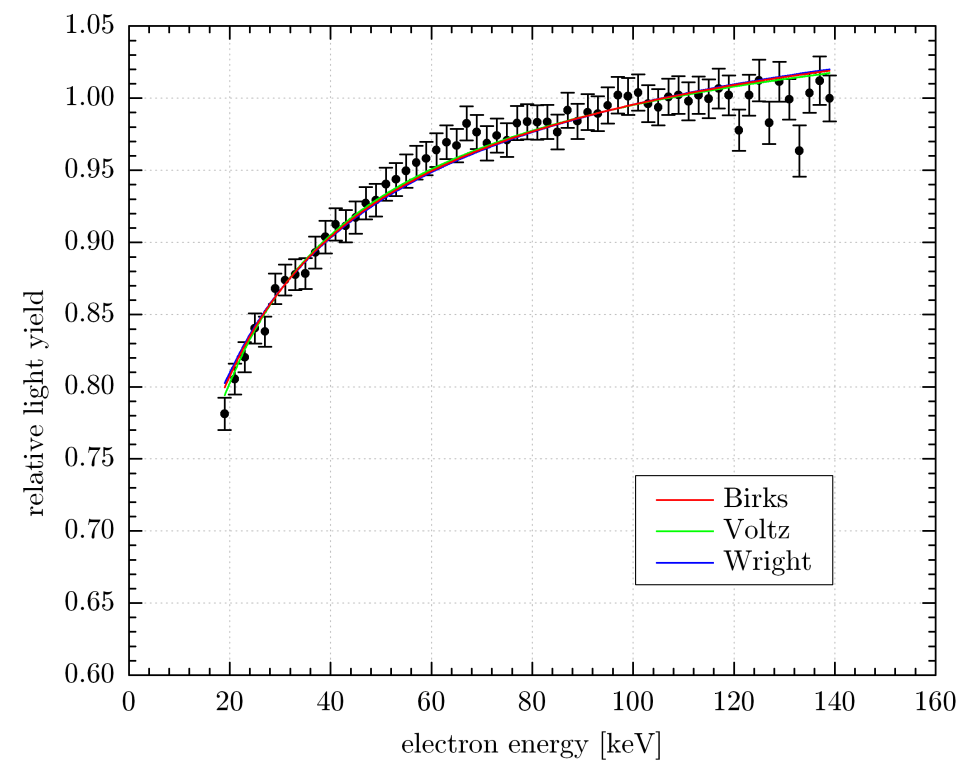

Figure 6. Relative deviation of the data points from linearity. The effect of quenching is now clearly visible for small electron energies. The plot displays the results for the DC $v$-Target scintillator. 


\section{Investigation of systematic errors}

The deviations from linearity due to quenching are small (figure 5). Therefore we performed systematic tests to ensure that no non-linearities are introduced by experimental artefacts. A detailed description of these investigations can be found in [10].

Germanium spectrometer. The germanium spectrometer was calibrated with a ${ }^{137} \mathrm{Cs}$ and a ${ }^{133} \mathrm{Ba}$ source, providing gammas of $662,384,356,303$ and $276 \mathrm{keV}$ energy. The pulse height spectrum was recorded for the two sources and the appearing peaks were associated with the gamma energies. A linear fit was then applied to the data points with its slope and offset defining the calibration curve. The linear relation between $E_{\gamma}^{\prime}$ and the spectrometer pulse heights was confirmed in our measurements. The relative deviation from linearity was smaller than $0.1 \%$. Additionally, the peak widths were used to determine the detector resolution. After the offline digital signal processing the energy resolution achieved was $3.92 \mathrm{keV}$ (FWHM) at $662 \mathrm{keV}$, which is about one ADC count.

The detector was calibrated twice for each measurement - before and after each run - to make sure that the calibration parameters do not change during a measurement.

Photomultiplier. The measurement of the PMT linearity was conducted in the MPIK's Faraday laboratory in an electromagnetically shielded darkroom. A laser diode emitting at $438 \mathrm{~nm}$ wavelength was pointed directly at the PMT's sensitive area. The laser was operated at $1.0 \%$ of its maximum power where its intensity is known to be sufficiently stable. Instead of changing the laser power, which could introduce a new source of errors, a set of calibrated neutral filters was used to reduce the light intensity. The filters were successively placed between the diode and the PMT, with their surfaces perpendicular to the laser ray. For each filter 1000 events were recorded. Between the runs with the filters in place, runs without any filters were performed.

For each intensity the PMT charge showed a narrow Gaussian distribution. Its mean value was plotted against the light intensity and the resulting curve showed a deviation from linearity $<1.0 \%$, which together with the standard deviation of the incident laser intensity of $0.4 \%$ (determined from the runs without filters), sums up to a total error of $<1.1 \%$.

Fan-in/out. To study the influence of the fan-in/out module we used a pulse generator. Short voltage pulses of $4 \mathrm{~ns}$ width and variable amplitude were fed into the fan module. The time between pulses was set to $1 \mu \mathrm{s}$. The signals before and after the fan were each recorded by the oscilloscope. As the fan-out is used for the PMT only, it is of interest if it reproduces the pulse integral, rather than the pulse height. Hence the signals were integrated. Input and output integrals showed a narrow Gaussian distribution and were fit to obtain the mean values. The fan-out and pulser means were then plotted against each other. The deviation from linearity was $<0.4 \%$ within the region of interest.

With the same setup the oscilloscope linearity could also be checked. For this, the pulser signals were directly connected to the oscilloscope. The recorded pulse heights were compared to the value adjusted on the pulse generator. The deviation from linearity measured was $<0.4 \%$. 
Discriminator. This experiment aims to take data for electrons of the lowest possible energies. The light yield of such events is just high enough that the PMT signals can still pass the discriminator threshold. However, the threshold may distort the event spectrum for these electron energies: variations of the baseline offset together with variations of the signal widths may lead to biases in the population above the threshold.

To perform a test of this effect, the threshold was lowered from $-20 \mathrm{mV}$ to $-70 \mathrm{mV}$ in steps of $10 \mathrm{mV}$ and the complete analysis procedure of the response function had to be done for each threshold level. The configuration of the setup was only altered in that just the $400 \mathrm{kBq}{ }^{137} \mathrm{Cs}$ source was used and placed directly under the cell. This test was conducted with the $v$-Target scintillator. For each discriminator threshold the $L(E)$-curve was obtained. These curves are identical for higher electron energies. But they separate at lower energies and the location of the branching depends on the threshold level. This means that the discriminator modifies the data points below the branching. In the same way it can be concluded that the data points are unaffected in the region where the curves are identical.

The locations of the branching points have then been obtained for curves of two consecutive thresholds, and compared with the values that one would have chosen directly from figure 3 . We took a conservative approach and concluded that it is safe to consider only energy slices where the complete peak lies above the threshold. For the threshold of $20 \mathrm{mV}$, which was used in the final measurement, this is the case for electron energies from $19 \mathrm{keV}$ upwards. Data points for lower energies were discarded in the analysis.

Spatial event distribution. To optimize light collection the full surface of one side of the quartz vial containing the scintillator sample is optically coupled to the PMT glass and centered to the photocathode. In all other directions the vial is surrounded by either the teflon block or the teflon cap on top providing almost $100 \%$ reflectivity for the scintillation light. The transparency of the liquid samples at the wavelenghts of scintillator emission is above $5 \mathrm{~m}$ which is very long compared to the dimensions of the quartz vial. In simulations rather constant light collection efficiency was found within the sample volume.

The spatial distribution of Compton electrons varies to some extent for different scattering angles. The distribution of the interactions was simulated for different electron energies. The simulations confirmed events are distributed in wide ranges of the small vial for a given energy and source position. In addition, data from the two source positions provide complementary distributions. So the systematic error related to the spatial distribution is expected to be negligible compared to the overall error.

The overall systematic errors quadratically added up to $1.2 \%$. This was taken as the systematic error of each data point and added quadratically to its statistical error.

\section{Results}

In the following we shortly present the models for $L(E)$ which were tested in our analysis. According to its definition in section 1, quenching depends largely upon the specific energy loss $d E / d x$ of the incident particle. It is consequently the principal quantity in the quenching models. In the case 
of incident electrons it is given by the Berger-Seltzer formula [11]

$$
\left(\frac{d E}{d x}\right)=2 \pi r_{e}^{2} n_{e} \frac{m_{e} c^{2}}{1-\gamma^{-2}}\left[\ln \left(2(\gamma+1) \frac{m_{e}^{2} c^{4}}{I^{2}}\right)+\frac{1}{\gamma^{2}}+\ln \frac{\tau^{2}}{4}+\frac{\tau^{2}}{8 \gamma^{2}}-\frac{2 \tau+1}{\gamma^{2}} \ln 2\right],
$$

with the electron rest energy $m_{e} c^{2}$, the classical radius of the electron $r_{e}$, the Lorentz factor $\gamma$ and $\tau=\gamma-1$. The electron density $n_{e}$ and the mean ionization energy $I$ of the medium can be calculated from the chemical composition of the scintillator and the mean ionization energies of the present elements, which are taken from [12].

The most prominent description of quenching is Birks' formula [13]

$$
\frac{d L}{d x}=\frac{L_{0}\left(\frac{d E}{d x}\right)}{1+k B\left(\frac{d E}{d x}\right)}
$$

in which $d L$ is the light produced when the particle travels the distance $d x, L_{0}$ is the light yield constant, and the parameter product $k B$ is generally treated as a single quenching parameter. It was originally developed for inorganic crystal scintillators, but the equation is widely used for all kinds of scintillators, even though the underlying physical processes are different. In cases where (4.2) is not precise enough, e.g. for incident heavy ions, it is often encountered in its generalized form

$$
\frac{d L}{d x}=\frac{L_{0}\left(\frac{d E}{d x}\right)}{1+A\left(\frac{d E}{d x}\right)+B\left(\frac{d E}{d x}\right)^{2}},
$$

with two quenching parameters $A$ and $B$, which was formulated by Chou [14].

The model proposed by Wright [15] makes a distinction between unimolecular and bimolecular quenching. Based on a rate equation, the combined effect of the two quenching mechanisms then leads to

$$
\frac{d L}{d x}=L_{0} \ln \left(1+B_{W}\left(\frac{d E}{d x}\right)\right)
$$

with $B_{W}$ being the quenching parameter in this model.

A detailed model by Voltz et al. [16] concentrates on processes on the molecular level. It includes the production of secondary electrons by the primary particle and distinguishes between molecules within the ionization column created by the primary particle and those far away from it. Additionaly, the argumentation includes a differentiation between singlet and triplet states. The different contributions by singlet and triplet states and secondary electrons sum up to

$$
\frac{d L}{d x}=L_{0}\left[A_{s}^{(1)}(1-F)\left(\frac{d E}{d x}\right) e^{-B_{s}(1-F) \frac{d E}{d x}}+F \cdot A_{s}^{(2)}\left(\frac{d E}{d x}\right)+A_{t}\left(\frac{d E}{d x}\right) e^{-B_{t} \frac{d E}{d x}}\right] .
$$

The quantities indexed with $t$ refer to triplet states, while those with $s$ refer to singlet states. The $B$ parameters are the quenching parameters, the $A$ parameters represent the light yield fractions from the different contributions, and $F$ is the fraction of electrons released with energies high enough to escape the ionization column. It was shown that $F$ depends explicitly on $z^{2}$, the square of the particle charge; a phenomenon which is not accounted for by (4.2) and (4.4), but was experimentally observed [17]. Equation (4.5) can be simplified for many practical purposes. A first simplification can be made if the contribution of triplet states is negligible. Furthermore, Voltz et al. argue 
that incident electrons do not create secondary electrons with energies high enough to escape the ionization column [18]. In this case $F$ would become zero and (4.5) reduces to

$$
\frac{d L}{d x}=L_{0}\left(\frac{d E}{d x}\right) \exp \left(-B\left(\frac{d E}{d x}\right)\right) .
$$

First we tested the suitability of the different quenching models (4.2), (4.4) and (4.6). For this analysis we wrote a computer program, which makes use of the Berger-Seltzer-formula (4.1) to numerically calculate the energy loss. The electron is repeatedly propagated in steps of $\Delta x=0.1 \mathrm{~nm}$ and $d E / d x$ is computed for this length and used as an input for the formulae (4.2), (4.4) and (4.6) to calculate the amount of light $\Delta L$ emitted in this step. The electron's kinetic energy is then reduced by $(d E / d x) \cdot \Delta x$. These steps are repeated until the electron comes to rest and the individual contributions $\Delta L$ are summed up to obtain the total amount of light $L$ created by an electron of initial energy $E$. The program repeats this procedure for each initial electron energy $E$ and produces the response function $L(E)$ predicted by the respective quenching model. It is important to note that (4.1) gives only the mean energy loss, but was considered here as a de-facto continuous energy loss of the electron in the scintillator (the influence of fluctuations in the energy loss or secondary particle creation is studied in the next section). A $\chi^{2}$-fit is then performed to find the best value for the quenching parameter. The fit results are shown in the figures 5 and 6 for the $v$-Target, and in the figures 7 and 8 for the GC. Table 2 summarizes the quenching parameters for all samples and for the different models.

For all scintillators and models the reduced $\chi^{2}$ yielded reasonable values. In the energy range investigated the $\chi^{2}$ values do not indicate a strong preference for a specific model and it is justified to use Birks' model for the Double Chooz data analysis. In the $v$-Target and GC the models predict higher values than experimentally measured at higher energies as well as below $30 \mathrm{keV}$ (figures 9 and 10).

The results can be explained qualitatively. The effectiveness of the energy transfer from solvent to fluor increases with the amount of the fluor until saturation occurs. The GC mixture with $2 \mathrm{~g} / \mathrm{l}$ PPO is the most affected by quenching. As the concentration of the primary fluor is low, the solvent-solute energy transfer is less effective and quenching can occur more often. From a comparison with the $5 \mathrm{~g} / \mathrm{l}$-version one can see experimentally that a higher fluor concentration reduces the influence of quenching as expected.

Though the PPO concentration of $1.5 \mathrm{~g} / 1$ in the Borexino scintillator (PC+PPO) is relatively small, the quenching parameter is smaller than for the GC mixtures. This is because the amount of PPO already lies above the critical concentration of this scintillator (see [19]). Hence the energy transfer between PC and PPO is very effective and quenching is small. Our result of the Birks parameter of this scintillator deviates from that given in [20] $(0.020 \mathrm{~cm} / \mathrm{MeV})$. This difference is most likely due to a different analysis technique used. The influence of different analysis methods on the quench parameter is discussed in the next section.

The smaller quench parameter in the $v$-Target is caused by the Gd-complex. This can be explained by the paths that the excitation energy can take. In a standard scintillator it mainly migrates to a fluor or is lost due to quenching. The presence of the non-scintillating metal organic Gdmolecules opens another transfer path [19]. The probability that a certain path is taken decreases with the total number of paths available. On one hand this reduces the overall light yield of the 


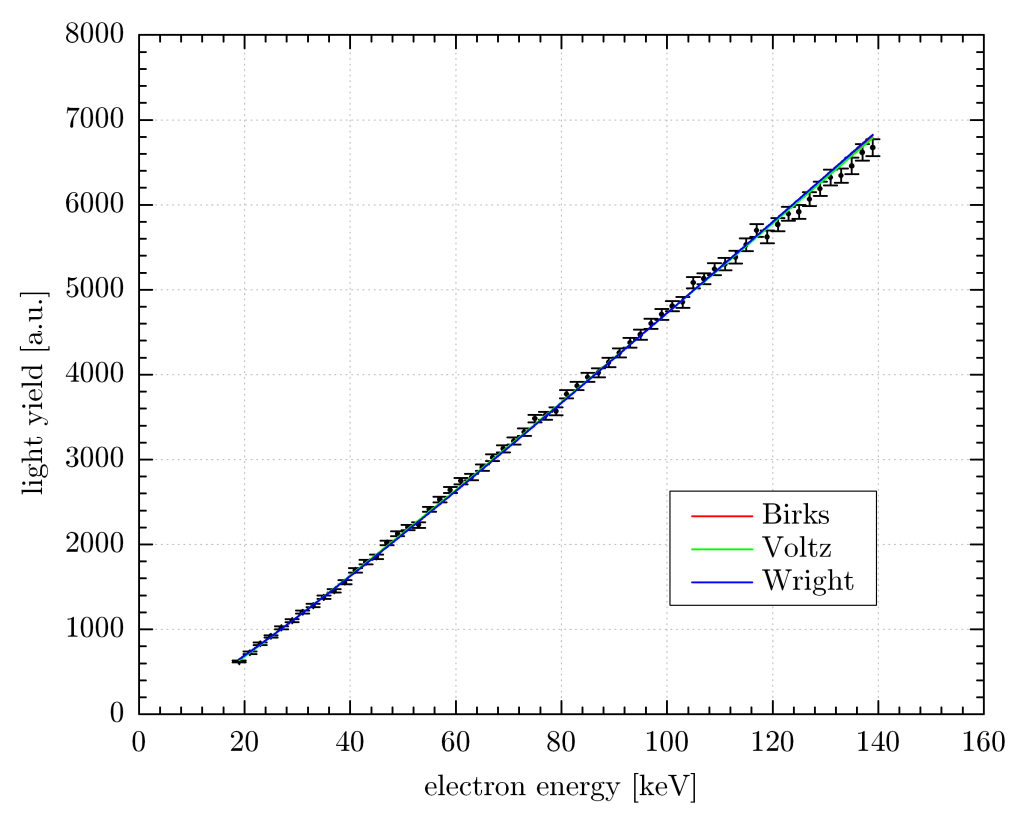

Figure 7. Experimental results for the DC Gamma Catcher ( $2 \mathrm{~g} / \mathrm{l} \mathrm{PPO}$ ) scintillator (linear scale).

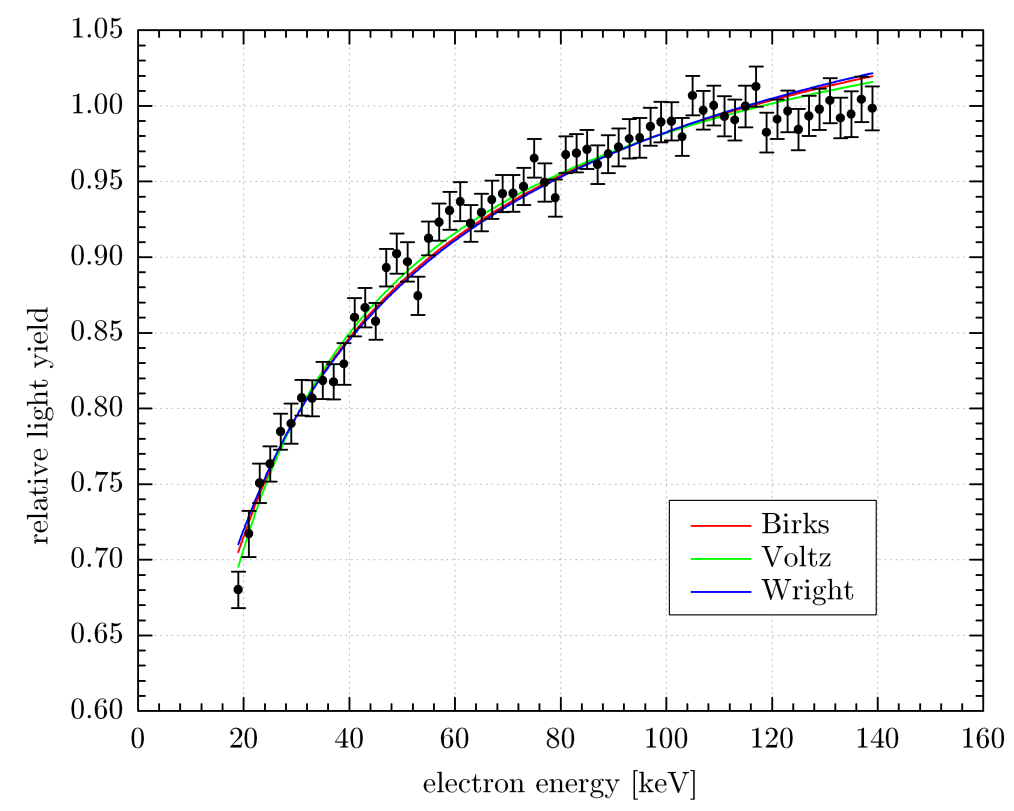

Figure 8. Experimental results for the DC Gamma Catcher (2 g/l PPO) scintillator (relative scale). 


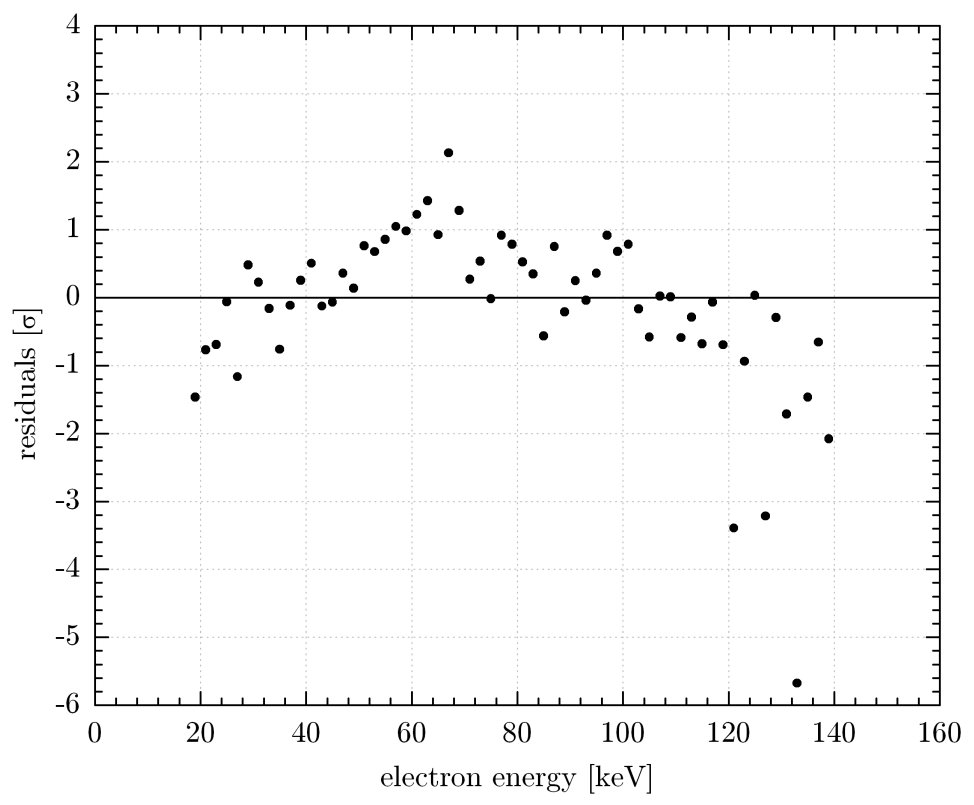

Figure 9. Residual plot for the DC $v$-Target scintillator.

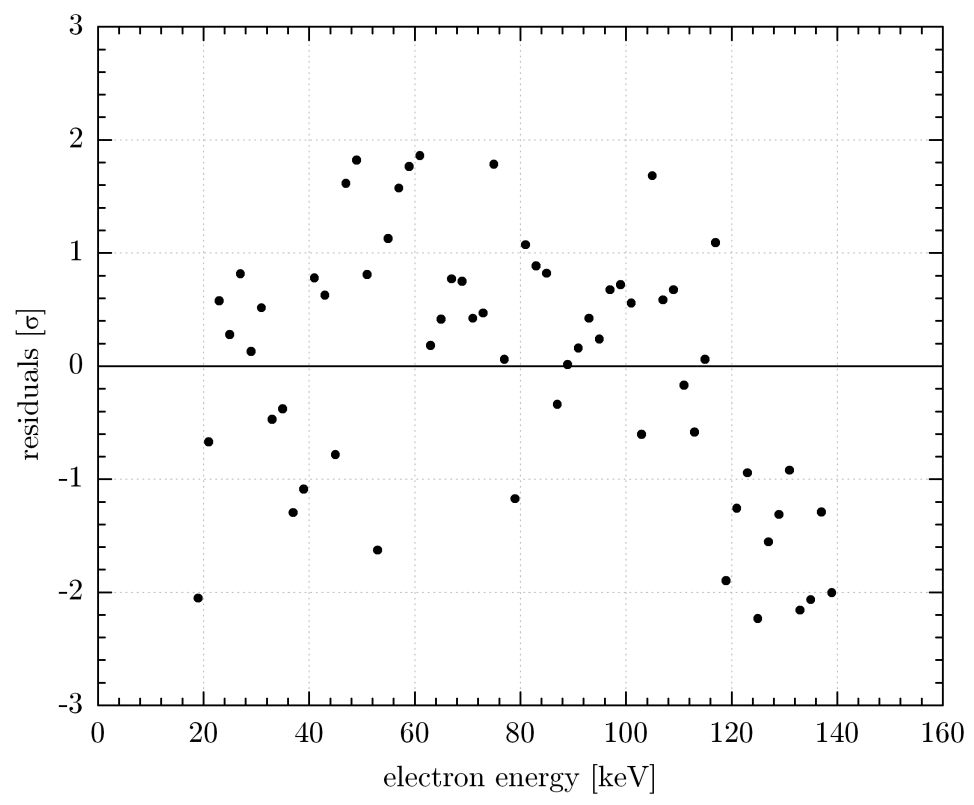

Figure 10. Residual plot for the DC Gamma Catcher (2 g/l PPO) scintillator. 
Table 2. Results for the quenching parameters of the different models, as obtained with the Berger-Seltzer formula. As the equations are of distinct mathematical forms, the quenching parameters cannot be directly compared between the models.

\begin{tabular}{|c|c|c|c|}
\hline Scintillator & Birks $[\mathrm{cm} / \mathrm{MeV}]$ & Voltz $[\mathrm{cm} / \mathrm{MeV}]$ & Wright $[\mathrm{cm} / \mathrm{MeV}]$ \\
\hline $\begin{array}{l}v \text {-Target } \\
\text { red. Chi square } \\
\text { (59 degrees of freedom) }\end{array}$ & $\begin{array}{l}0.01582_{-0.00059}^{+0.00058} \\
0.78\end{array}$ & $\begin{array}{l}0.01216 \pm 0.00038 \\
0.66\end{array}$ & $\begin{array}{l}0.0370 \pm 0.0015 \\
0.89\end{array}$ \\
\hline $\begin{array}{l}\mathrm{GC}(2 \mathrm{~g} / 1 \mathrm{PPO}) \\
\text { red. Chi square } \\
(59 \text { degrees of freedom })\end{array}$ & $\begin{array}{l}0.02787_{-0.00087}^{+0.00091} \\
0.97\end{array}$ & $\begin{array}{l}0.01977 \pm 0.00045 \\
0.84\end{array}$ & $\begin{array}{l}0.0733 \pm 0.0025 \\
1.29\end{array}$ \\
\hline $\begin{array}{l}\text { GC }(5 \mathrm{~g} / 1 \text { PPO }) \\
\text { red. Chi square } \\
\text { (59 degrees of freedom) }\end{array}$ & $\begin{array}{l}0.02452_{-0.00078}^{+0.00081} \\
0.98\end{array}$ & $\begin{array}{l}0.01755_{-0.00046}^{+0.00045} \\
0.88\end{array}$ & $\begin{array}{l}0.0605_{-0.0022}^{+0.0023} \\
1.08\end{array}$ \\
\hline $\begin{array}{l}\mu \text {-Veto } \\
\text { red. Chi square } \\
\text { ( } 59 \text { degrees of freedom) }\end{array}$ & $\begin{array}{l}0.01519_{-0.00057}^{+0.00059} \\
0.88\end{array}$ & $\begin{array}{l}0.01170_{-0.00037}^{+0.00038} \\
0.82\end{array}$ & $\begin{array}{l}0.03553 \pm 0.0015 \\
0.93\end{array}$ \\
\hline $\begin{array}{l}\mathrm{PC}+\mathrm{PPO} \\
\text { red. Chi square } \\
\text { ( } 59 \text { degrees of freedom) }\end{array}$ & $\begin{array}{l}0.01715_{-0.00057}^{+0.00059} \\
0.77\end{array}$ & $\begin{array}{l}0.01272_{-0.00035}^{+0.00036} \\
0.99\end{array}$ & $\begin{array}{l}0.0412_{-0.0015}^{+0.0016} \\
0.67\end{array}$ \\
\hline $\begin{array}{l}\text { plastic scintillator } \\
\text { red. Chi square } \\
\text { ( } 54 \text { degrees of freedom) }\end{array}$ & $\begin{array}{l}0.01529_{-0.00070}^{+0.00068} \\
0.41\end{array}$ & $\begin{array}{l}0.01174 \pm 0.00045 \\
0.41\end{array}$ & $\begin{array}{l}0.0358 \pm 0.0018 \\
0.42\end{array}$ \\
\hline
\end{tabular}

mixture, since the energy transfer to PPO has to compete with the transfer to the Gd-complex. On the other hand it also reduces the influence of quenching, as it has to compete with the Gd-complex as well. Its relative influence is therefore smaller than in mixtures where there is only one path. A second reason for the small quenching parameter is the absence of mineral oil in the $v$-Target. Admixtures of mineral oil are expected to increase quenching since the oil molecules are less effective in the energy transfer. From the small value of the quenching parameter in the $\mu$-Veto scintillator we expect that the energy transfer path from LAB to PPO is very effective.

\section{Monte Carlo tuning}

The experimental results presented above were used to tune the Monte Carlo (MC) simulation of the Double Chooz experiment. It turned out that the MC settings influence the energy dependence of the scintillation light. It is possible to compensate for this effect by applying effective $k B$ values in the simulation, which can be different from the $k B$ obtained by the numerical method from section 4 . Here we use the $v$-Target scintillator as an example to illustrate the effect.

Monte Carlo model. The MC software uses the quenching model originally developed by Birks. Particles are propagated step by step. For each step the energy loss of the particle in a given medium is calculated with fluctuations drawn from a straggling function. If the energy loss in a given step 


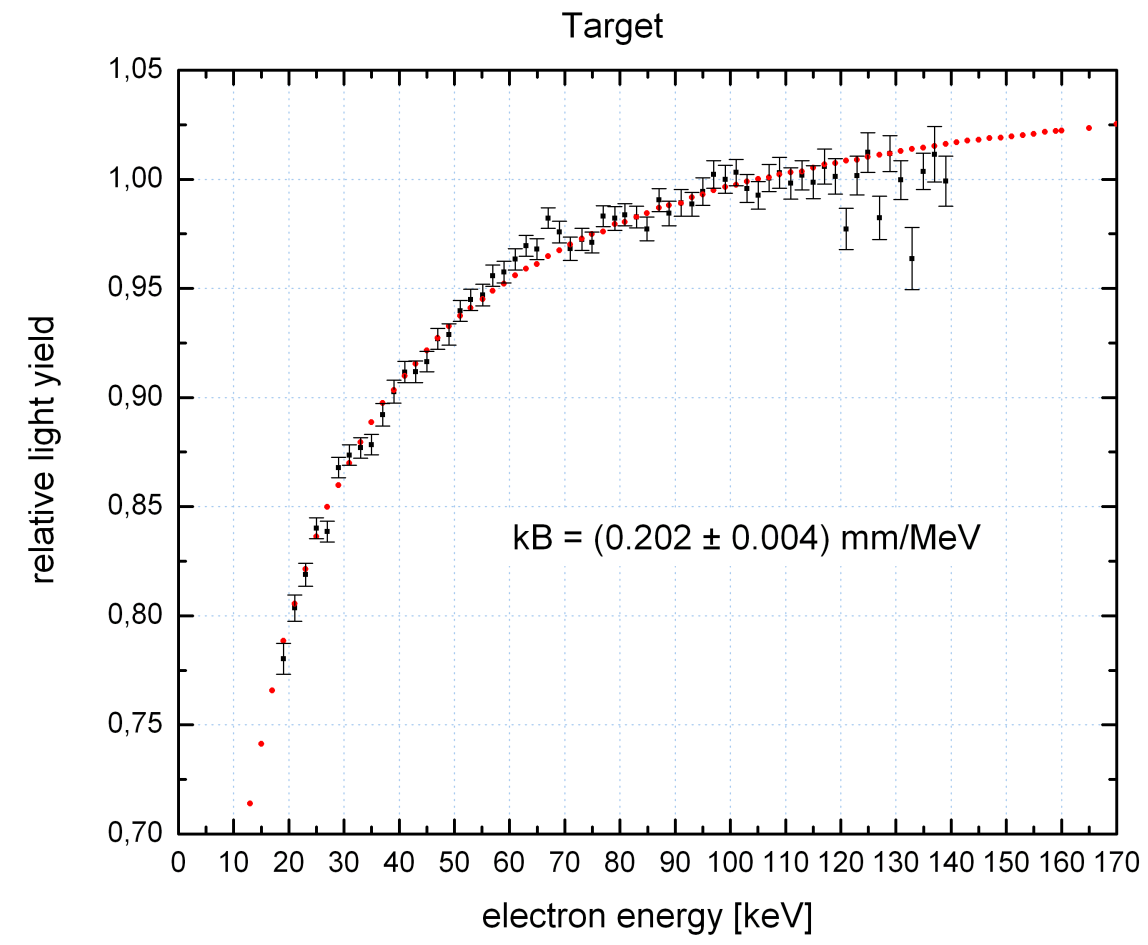

Figure 11. Best fit of Monte Carlo light production (red points) to data for the DC $v$-Target scintillator. Here the default parameters for the stepping commands have been used. We only included statistical errors.

exceeds a certain value a secondary particle is explicitly generated. The user can control the step size and specify the threshold for explicit secondary production. One can also enable or disable the fluctuations.

The energy lost by the primary particle in a given step is $\Delta E_{0}$. In the approach described here, which is also used in the GEANT4 class G4EmSaturation by default, the number of scintillation photons generated is proportional to the quantity $\Delta E_{q}$ which is called quenched energy (or visible energy). This quantity is calculated using an equation which corresponds to (4.2):

$$
\Delta E_{q}=\frac{\Delta E_{0}}{1+k B\left(\frac{\Delta E_{0}}{\Delta x}\right)} .
$$

Here $\Delta x$ is the length of the considered step and $k B$ is the quenching constant. The equation for the energy loss of electrons used by Geant $4{ }^{9}$ is the Berger-Seltzer equation (4.1).

Fit procedure. For multiple $k B$ values and for each energy for which an experimental data point is available we simulated 1000 electrons. This guarantees that the statistical error on the mean of the simulated distribution of $E_{q}$ is small. Then we did a $\chi^{2}$ fit of the data with the simulated mean $E_{q}$ values with two free parameters: one global normalization and $k B$. The shape of the function determines the best fit value of $k B$ which is then used in our MC simulation. The fit for the default settings in GEANT4 is shown in figure 11.

\footnotetext{
${ }^{9}$ For this analysis Geant4.9.2p02 was used.
} 
Table 3. Results for $k B$ with different stepping commands for the $v$-Target scintillator. The entries in the table are the parameter(s) of the corresponding command. The statistical errors of the fits are given.

\begin{tabular}{|lllll|}
\hline /process/eLoss/StepFunction & /run/particle/setCut & /process/eLoss/fluct & $k B[\mathrm{~cm} / \mathrm{MeV}]$ \\
\hline 0.20 & $1 \mathrm{~mm}$ (default) & $0.01 \mathrm{~mm}$ (default) & true (default) & $0.0202 \pm 0.0003$ \\
0.05 & $0.1 \mathrm{~mm}$ & $0.01 \mathrm{~mm}$ & true & $0.0232 \pm 0.0004$ \\
0.05 & $0.001 \mathrm{~mm}$ & $0.01 \mathrm{~mm}$ & true & $0.0174 \pm 0.0003$ \\
0.05 & $10^{-5} \mathrm{~mm}$ & $0.01 \mathrm{~mm}$ & true & $0.0107 \pm 0.0003$ \\
0.05 & $10^{-5} \mathrm{~mm}$ & $10^{5} \mathrm{~mm}$ & false & $0.0164 \pm 0.0003$ \\
0.20 & $1 \mathrm{~mm}$ & $0.001 \mathrm{~mm}$ & true & $0.0287 \pm 0.0005$ \\
\hline
\end{tabular}

Stepping control commands. There are several commands available to control the stepping process. The user can control the step length using two parameters p1 and p2 with /process/eLoss/StepFunction p1 p2. The first parameter 1 specifies which fraction of the stopping range a particle can travel at most within one step. Secondly, a minimal step length can be specified (this is needed to guarantee that the stepping process comes to an end). We also studied the variation of the threshold for explicit secondary particle generation with the command /run/particle/setCut p3. If the energy loss in a single step for a chosen physics process is high enough that the particle generated in this process has a range above the threshold $\mathrm{p} 3$ then the particle is explicitly generated and tracked by GEANT4. A lower threshold means that we get more secondary particles. For these secondary particles the energy loss for one step is calculated in the same way as for the primary particle and $\Delta E_{q}$ for this step is added to the total $E_{q}$. The effect of switching off fluctuations in the energy loss calculation was also studied with the command /process/eLoss/fluct false.

Results. In section 4 we presented the $k B$ parameters obtained by the analysis not using the MC software. There we integrated (4.2) numerically with small step sizes $\Delta x$. We did not explicitly calculate the energy loss for secondary particles and we did not include fluctuations. As a check of the consistency of this method with the MC tuning we can use the commands described above to check if we arrive at the same result with MC if we apply similar conditions: we chose a maximal relative energy loss per step of $5 \%$ and a minimal step length of $10^{-5} \mathrm{~mm}$ to approach the small step size in the numerical analysis. No secondary particles were produced (a very high threshold for secondary particle production was chosen) and we turned off fluctuations. For the $v$-Target scintillator the result for $k B$ is $(0.0164 \pm 0.0003) \mathrm{cm} / \mathrm{MeV}$ and thus matches the result of the numerical method. Note that for the studies in this section we only consider the statistical errors and did not include the $1.2 \%$ of systematical error for each data point. This explains why the error intervals are generally smaller than the ones in section 4 . Best fit values of $k B$ for several stepping commands are summarized in table 3 .

Figure 11 shows the resulting best fit for the default parameters. For these settings $\chi^{2}$ of the fit to the experimental data is lowest of all the options we tested, even so the number of steps is small. One can see that the best fit values for $k B$ changed more than a factor of two when we compared different settings in Geant 4 . This leads us to the conclusion that the $k B$ parameters as extracted in section 4 can only be used in MC if the simulation settings are in agreement with the 
assumptions of the analysis. For a wide range of parameter settings the data can be well described in the available energy range by using an effective Birks parameter.

\section{Conclusion}

In this work we studied ionization quenching on five liquid scintillator compositions and one plastic scintillator. Three common mathematical models were tested for suitability and all three formulae were able to reproduce the experimental data. Within the precision of the measurement there was no clear preference for any of the models described above. So we chose Birks' model for the analysis in Double Chooz as it is the simplest and most common one.

The experimental data obtained was also used as an input paramter in the Monte Carlo simulation of Double Chooz. It was found that the specific simulation settings have an impact on the non-linearity of the scintillation light production in the $\mathrm{MC}$ and it could be concluded that the $k B$ has to be newly determined to compensate for changes in the settings. Tabulated values of the quenching parameter should also give the assumptions of the analysis method which was used to obtain it.

\section{Acknowledgments}

We would like to thank the members of the Double Chooz collaboration, and especially the DC Offline Working Group, for their support and fruitful discussions on this topic. Thanks are also due to the central mechanical workshop at the MPIK for the production of mechanical parts used in our experiment. We are grateful to Alexander Tadday and the CALICE group at the Universität Heidelberg for providing us with a sample of the plastic scintillator. F.X. Hartmann thanks the Max-Planck-Society for financial support under a research contract to Hartmann Scientific, an independent professional research entity licensed in the City of Virginia Beach, Virginia, USA.

\section{References}

[1] Double Chooz collaboration, F. Ardellier et al., Double Chooz: A search for the neutrino mixing angle theta(13), hep-ex/0606025.

[2] C. Buck, F.X. Hartmann, S. Schönert and U. Schwan, Development of an optically pure In $\beta$-diketonate for the scintillator of an ${ }^{115}$ In-loaded solar neutrino detector, J. Radioanal. Nucl. Ch. 258 (2003) 255.

[3] C. Buck, F.X. Hartmann, T. Lasserre, D. Motta, S. Schönert and U. Schwan, Luminescent properties of a new In-based liquid scintillator system, J. Lumin. 106 (2004) 57.

[4] D. Motta, C. Buck, F.X. Hartmann, T. Lasserre, S. Schönert et al., Prototype scintillator cell for an In-based solar neutrino detector, Nucl. Instrum. Meth. A 547 (2005) 368 [physics/0502086].

[5] C. Buck, F.X. Hartmann, D. Motta and S. Schönert, Energy transfer and light yield properties of a new highly loaded indium(III) $\beta$-diketonate organic scintillator system, Chem. Phys. Lett. 435 (2007) 255.

[6] F.X. Hartmann, Organic liquid scintillators for low $v$ detection, presentation at LowNu 2002: Third International Workshop on Low Energy Solar Neutrinos, Heidelberg, Germany, 2002. 
[7] C. Buck, F.X. Hartmann, S. Schönert and U. Schwan, Development of an optically pure $G d$-beta-diketonate for the liquid scintillator of anti-neutrino detectors, article in preparation for $J$. Radioanal. Nucl. Ch. .

[8] J.B. Birks, The theory and practice of scintillation counting, Pergamon Press, New York, 1964.

[9] M-N. Péron and P. Cassette, Mesure de la réponse lumineuse de scintillateurs liquides à des électrons mono-énergétiques d'énergie inférieure à $100 \mathrm{keV}$, Bulletin du BNM 105 (1996) 33.

[10] S. Wagner, Ionization quenching by low energy electrons in the Double Chooz scintillators, diploma thesis, Ruprecht-Karls-Universität Heidelberg (2010).

[11] S.M. Seltzer and M.J. Berger, Evaluation of the collision stopping power of elements and compounds for electrons and positrons, Int. J. Appl. Rad. Isot. 33 (1982) 1189.

[12] International Commission on Radiation Units and Measurements, Stopping powers for electrons and positrons, ICRU Report 37, Bethesda (1984).

[13] J.B. Birks, Scintillations from organic crystals: specific fluorescence and relative response to different radiations, Proc. Phys. Soc. A 64 (1951) 874.

[14] C.N. Chou, The nature of the saturation effect of fluorescent scintillators, Phys. Rev. 87 (1952) 904.

[15] G.T. Wright, Scintillation response of organic phosphors, Phys. Rev. 91 (1953) 1282.

[16] R. Voltz and G. Laustriat, Radioluminescence des milieux organiques I. Étude cinétique, J. Phys. France 29 (1968) 159.

[17] R. Voltz, H. Dupont and G. Laustriat, Radioluminescence des milieux organiques. II. vérification expérimentale de l'étude cinétique, J. Phys. France 29 (1968) 297.

[18] R. Voltz, J. Lopes da Silva, G. Laustriat and A. Coche, Influence of the nature of ionizing particles on the specific luminescence of organic scintillators, J. Chem. Phys. 45 (1966) 3306.

[19] C. Aberle, C. Buck, F.X. Hartmann and S. Schönert, Light yield and energy transfer in a new Gd-loaded liquid scintillator, Chem. Phys. Lett. 516 (2011) 257.

[20] W. Maneschg, Low-energy solar neutrino spectroscopy with Borexino: Towards the detection of the solar pep and CNO neutrino flux, $\mathrm{PhD}$ thesis, Ruprecht-Karls-Universität Heidelberg (2011).

[21] GEANT4 collaboration, S. Agostinelli et al., GEANT4: A Simulation toolkit, Nucl. Instrum. Meth. A 506 (2003) 250. 